\title{
Fibroma ossificante complicado com piocele de seios paranasais: um relato de caso
}

\section{Ossificant fibroma associeted with frontal sinus' piocele: a case report}

Gabriela de Andrade Meireles Bezerra ${ }^{1,2}$. Yasmine Maria Leódido Fortes ${ }^{2}$. Clara Mota Randal Pompeu ${ }^{1,2}$. Jéssica de Castro Vidal Sousa ${ }^{1,2}$.Viviane Carvalho da Silva ${ }^{1,2}$. André Alencar Araripe Nunes ${ }^{1,2}$.

1 Hospital Universitário Walter Cantídio (HUWC/UFC/EBSERH), Fortaleza, Ceará, Brasil. 2 Universidade Federal do Ceará (UFC), Fortaleza, Ceará, Brasil.

\section{RESUMO}

Objetivos: Apresentar um caso incomum de recidiva de fibroma ossificante complicado com osteomielite e piocele de seio frontal. Relato de caso: Paciente, 50 anos, com diagnóstico prévio de fibroma ossificante, tendo sido submetido a procedimento cirúrgico para excisão do tumor em face há 23 anos. Evoluiu há cerca de 1 ano com o aparecimento de orifícios fistulosos em face, com drenagem de secreção purulenta. Realizou tomografia computadorizada de seios paranasais que evidenciou a presença de trajetos fistulosos cutâneos em região frontal esquerda e frontotemporal direita, onde se evidencia pequena coleção abscessulizada adjacente ao plano meníngeo. O tratamento incluiu um acesso externo em face para a drenagem de coleção purulenta, além de antibioticoterapia prolongada. Conclusão: O fibroma ossificante de seios paranasais é de ocorrência rara. Seu tratamento cirúrgico é, muito frequentemente, desafiador para o cirurgião devido à proximidade com estruturas nobres, como encéfalo e órbita. Faz-se necessário o envolvimento de uma equipe multidisciplinar para um seguimento adequado dos pacientes e manejo das possíveis complicações pós-cirúrgicas.

Palavras-chave: Seios paranasais. Endoscopia. Fibroma ossificante.

\section{ABSTRACT}

Objectives: To present an unusual case of recurrent ossifying fibroma complicated with osteomyelitis and frontal sinus piocele. Case report: A 50-year-old patient with a previous diagnosis of ossifying fibroma who underwent a surgical procedure for tumor excision in the face 23 years ago. He evolved, about 1 year ago, with the appearance of facial fistulous orifices, with drainage of purulent discharge. The patient underwent paranasal sinuses computed tomography, which showed the presence of cutaneous fistulous pathways in the left frontal and right frontotemporal region, where a small abscessulized collection adjacent to the meningeal plane was notted. The treatment included an external face access, for the drainage of the purulent collection, and prolonged antibiotic therapy. Conclusion: Paranasal sinus ossifying fibroma is a rare disease. Its surgical treatment is very often challenging for the surgeon due to its proximity to noble structures such as the brain and orbit. The involvement of a multidisciplinary team is necessary for proper patient follow-up and management of postoperative complications.

Keywords: Paranasal sinuses. Endoscopy. Fibroma, ossifying.

Autor correspondente: Gabriela de Andrade Meireles Bezerra, Rua Nunes Valente, 1250, Aldeota, Fortaleza, Ceará. CEP: 60125-035. Telefone: +55 85 99910-2249. E-mail: gabrielameirelesb@gmail.com

Conflito de interesses: Não há qualquer conflito de interesses por parte de qualquer um dos autores.

Recebido em: 29 Ago 2019; Revisado em: 09 Ago 2020; Aceito em: 06 Jul 2021. 


\section{INTRODUÇÃO}

O fibroma ossificante (FO) constitui uma lesão fibro-óssea benigna rara, localmente agressiva, que ocorre principalmente em ossos craniofaciais. ${ }^{1}$ A sua etiologia ainda é desconhecida, porém, sugere-se que alterações odontogênicas, de desenvolvimento e traumas possam estar associados com o seu desenvolvimento. ${ }^{2}$ É encontrado na mandíbula em 75 a $89 \%$ dos casos, na maxila em $10 \%$ e tem rara ocorrência em órbita e seios paranasais. ${ }^{3}$ FO tem maior prevalência entre mulheres, principalmente entre a segunda e quarta décadas de vida. FO de seios paranasais são principalmente encontrados em mulheres afroamericanas e entre a terceira e quarta décadas de vida. ${ }^{4}$ Histologicamente são caracterizados pela substituição de osso normal por tecido fibroso, contendo quantidades variáveis de material mineralizado e cemento. ${ }^{4}$ Geralmente as lesões apresentam um crescimento lento e não causam sintomas significativos, podendo ser diagnosticados incidentalmente por exames de imagem. Após longos períodos de crescimento silente, entretanto, o tumor pode apresentar grandes dimensões e gerar compressão de estruturas anatômicas adjacentes. O sintoma mais encontrado é edema facial, seguido por obstrução nasal, cefaleia, proptose e diplopia. ${ }^{1}$ Podem ocorrer complicações como mucoceles, infecções de sistema nervoso central e redução da acuidade visual. ${ }^{5} \mathrm{O}$ tratamento cirúrgico é o de escolha. O presente estudo objetiva compartilhar um caso incomum de recidiva fibroma ossificante complicado com osteomielite e piocele de seio frontal.

\section{RELATO DE CASO}

Paciente, sexo masculino, 50 anos, com diagnóstico prévio de fibroma ossificante, tendo sido submetido a excisão de tumor em face, no ano de 1996, em outro serviço. Permaneceu assintomático até março de 2018, quando se iniciou a drenagem de secreção purulenta por três orifícios fistulosos em face (Figura 1), em topografia de região frontal à direita, região frontotemporal direita e de região periorbitária medial à direita. Paciente negava febre ou qualquer outro sintoma constitucional. Nasofibroscopia realizada na admissão evidenciou sinais de sinusotomia maxilar, etmoidal e esfenoidal à direita, com presença de secreção purulenta e crostas em recesso esfenoetmoidal. A tomografia computadorizada (TC) de seios paranasais demonstrou cavidade frontal alargada, com paredes escleróticas espessadas, contendo material heterogêneo em seu interior, estendendo-se para células etmoidais, paredes mediais da órbita e seio maxilar direito. Aparente sequestro ósseo. Presença de trajetos fistulosos cutâneos em região frontal esquerda e frontotemporal direita, onde se evidencia pequena coleção purulenta adjacente ao plano meníngeo (Figura 2). Paciente foi submetido a drenagem cirúrgica de coleção purulenta de face por meio de acesso externo ao seio frontal direito (Figura 3), com remoção de sua tábua anterior, de etmoide e de osso nasal à direita, com comunicação nasal. Foi observado no intraoperatório abundante secreção purulenta, além da presença de paquimeninge, com fístula posterior, sendo realizada a correção da fístula liquórica. A biópsia do material cirúrgico revelou displasia fibrosa associada a osteomielite aguda. O resultado da cultura foi positivo para
Klebsiella pneumoniae e Pseudomonas aeruginosa, tendo iniciado antibioticoterapia dirigida (cefepime e clindamicina), que foi mantida por período prolongado. O paciente evoluiu estável e sem queixas. Ressonância magnética de crânio realizada pós-cirurgia demonstrou a presença de herniação do parênquima encefálico e meninges através da cavidade cirúrgica. Foi decidido acompanhamento ambulatorial para posterior abordagem conjunta da neurocirurgia com otorrinolaringologia para correção de herniação. O paciente também foi encaminhado para o serviço de cirurgia bucomaxilo-facial para planejamento de posterior reconstrução de falha óssea em face pós-procedimento cirúrgico (Figura 4).

Figura 1. Orifícios fistulosos em face com drenagem de secreção purulenta.

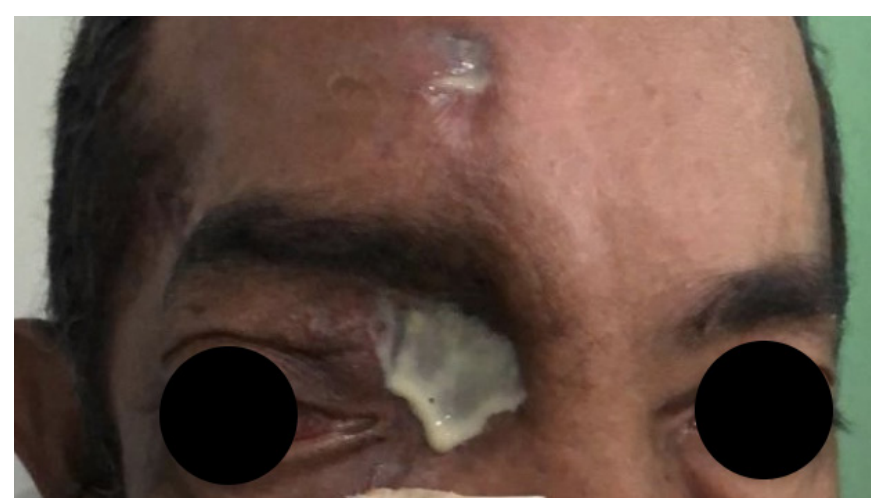

Figura 2. Corte axial de TC de seios paranasais, mostrando lesão fibro-óssea comprimindo região orbitária à direita e fístula entre cavidade nasal e pele.

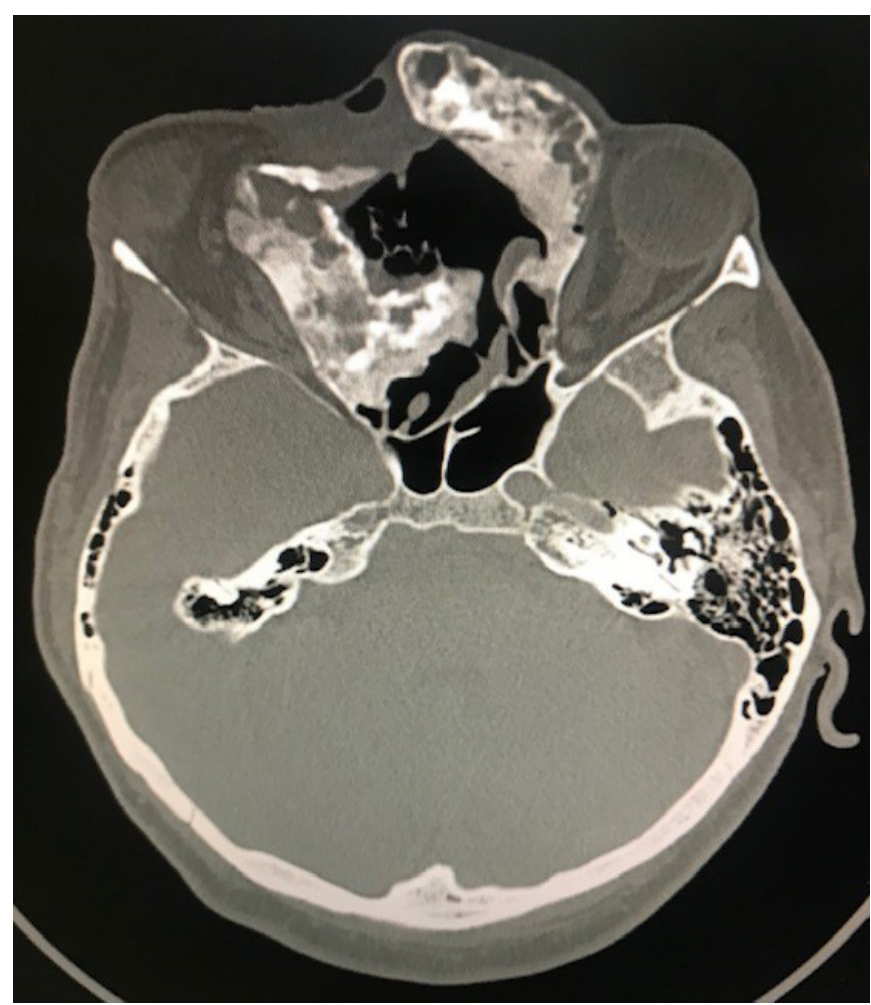


Figura 3. Acesso externo ao seio frontal.

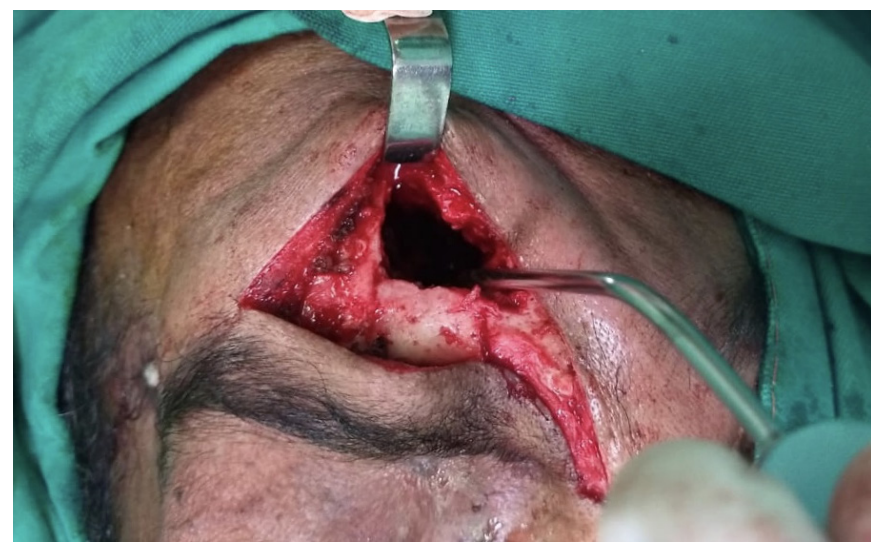

Figura 4. Paciente em retorno de 3 meses pós cirurgia.

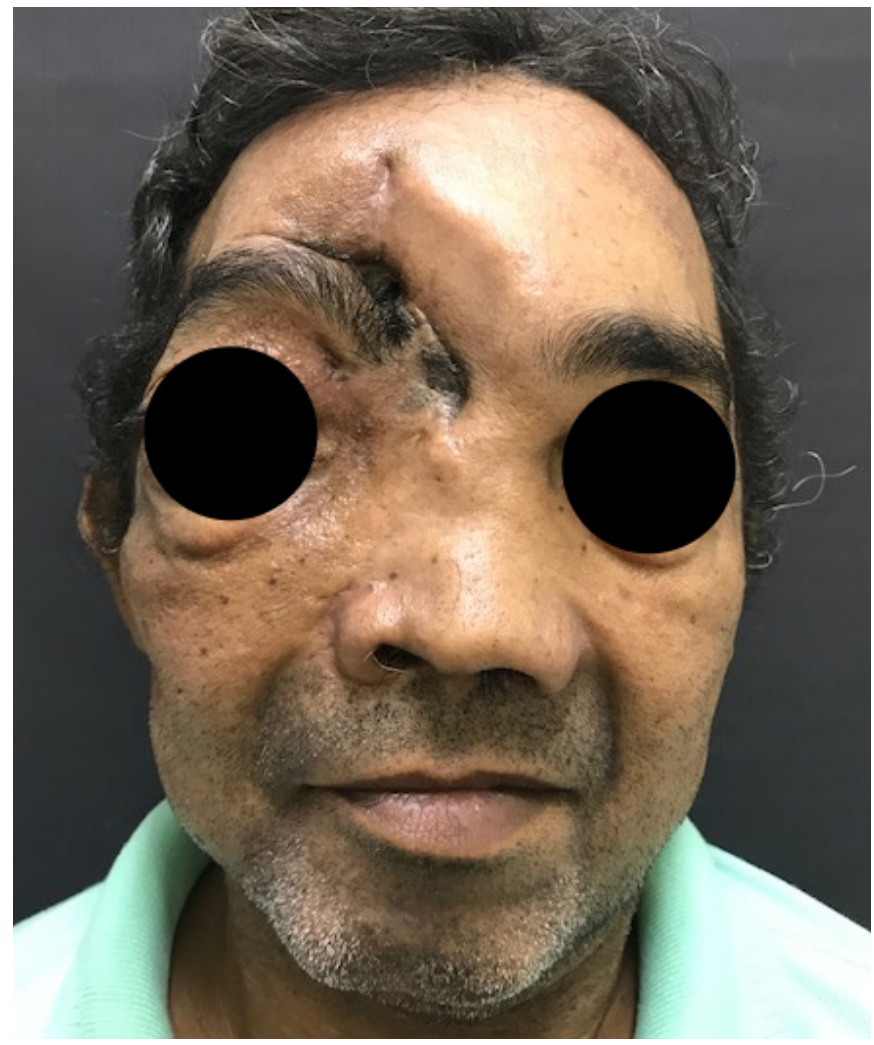

\section{DISCUSSÃO}

O caso apresentado demonstra sua relevância devido a sobreposição de uma doença óssea de ocorrência pouco frequente, com o processo infeccioso extenso dos seios frontais e etmoidais, além da presença de fístula liquórica associada, tendo o paciente, a despeito do quadro exposto, evolução favorável.

O tratamento cirúrgico da mucocele é o de escolha e pode ser realizado por via endoscópica isolada, transfacial, transoral ou por craniotomia, porém, na maior parte dos casos, uma abordagem via técnica aberta é necessária para a visualização completa da lesão, podendo permitir sua ressecção completa., ${ }^{3,5}$ Não há evidências na literatura de que a abordagem endoscópica é superior à aberta, ou viceversa. ${ }^{5}$ A radioterapia é contraindicada, pois pode aumentar a possibilidade de transformação maligna tumoral. ${ }^{6}$ Embora não há relatos na literatura de transformação maligna de fibroma ossificante. ${ }^{1}$ Taxas de recorrência variam de 30 a 58\%. ${ }^{7}$ A localização atípica da lesão no complexo frontoetmoidal, apresentada no caso, causa diversos desafios durante o ato cirúrgico, havendo dificuldades na sua excisão completa, devido à proximidade com estruturas nobres, como cérebro e globo ocular. ${ }^{7}$ Até o final da década de 1980, a abordagem via aberta, com ampla incisão externa era a única abordagem usada. A incisão em seio topografia de seio frontal permitia acesso ao seio frontal e complexo etmoidal. Atualmente, porém, a via endoscópica é bem mais difundida e, muitas vezes, preferida no momento de acesso a tais seios da face. Essa via de abordagem pode favorecer uma melhor visualização da área abordada. No caso apresentado, já havia comprometimento extenso do tecido ósseo da região frontal, com sequestro ósseo. A abordagem via externa foi preferida devido ao comprometimento do tecido ósseo, que dava acesso ao seio frontal, e à presença de coleção purulenta abundante no local. Dessa maneira, houve melhor exposição da área infectada, sua limpeza e remoção de tecidos desvitalizados. A presença de mucoceles pode gerar defeitos durais e expansão intradural. ${ }^{8}$ Fístulas liquóricas são complicações descritas e podem ser reparadas no intraoperatório, como ocorreu com o paciente citado. ${ }^{5}$

A presença de osteomielite em concomitância com o quadro de fibroma ossificante é descrita como incomum na literatura, podendo relacionar-se com a vascularização diminuída na massa tumoral, tornando o tecido ósseo mais susceptível a infecção. ${ }^{9}$

\section{CONCLUSÃO}

O fibroma ossificante de seios paranasais é de ocorrência rara. Seu tratamento cirúrgico é, muito frequentemente, desafiador para o cirurgião devido à proximidade com estruturas nobres, como encéfalo e órbita. Faz-se necessário o envolvimento de uma equipe multidisciplinar para um seguimento adequado dos pacientes e manejo das complicações pós-cirúrgicas.

\section{REFERÊNCIAS}

1. Alghonaim Y, Alhhumaid S, Arafat A. Aggressive ossifying fibroma of right ethoidal sinus: a case report. Int J Surg Case Rep. 2018;53:513-6.
2. Charavarty S, Datta D, Boro E, Dutta B. Ossifying fibroma in the sinonasal tract: a rare case report. J Clin Diagn Res. 2014;8:9-10. 
3. Baumann I, Zimmermann R, Dammann F, Maassen M. Ossifying fibroma of the ethmoid involving the orbit and the skull base. Otolaryngol Head Neck Surg. 2005;133:158-9.

4. Liu J, Thompson L, Janisiewicz A, Shibuya T, Keschner D, Garg $\mathrm{R}$, et al Ossifying fibroma of the maxilla and sinonasal tract: case series. Allergy Rhinol. 2017;8:32-6.

5. Manes R, Ryan M, Batra P, Mendelsohn D, Fang Y, Marple B. Ossifying fibroma of the nose and paranasal sinuses. Int Forum Allergy Rhinol. 2012;3:161-8.

6. Jurlina M, Skitareli N, Passali D, Passali F, Mladina R. Endonasal endoscopic resection of ossifying fibroma involving the ethmoid sinus, orbit and anterior skull base: case report and literature review. Acta Otorhinolaryngol Ital. 2016;36:144-8.

7. Laxamana J, Gutierrez R, Claridad R. Giant ossifying fibroma of the frontoethmoid sinus: a silent peril. Philipp J Otolaryngol Head Neck Surg. 2013;28:22-5.

8. Manaka H, Tokoro K, Sakata K, Ono A, Yamamoto I. Intradural extension of mucocele complicating frontoethmoid sinus osteoma: case report. surg neurol. 1998;50:453-6.

9. Punyani S, Jasuja V, Kumar P, Kaushik R. Massive expansile ossifying fibroma of the mandible with osteomyelitis. Oral Radiology Springer. 2014;3:243-8.

\section{Como citar:}

Bezerra GA, Fortes YM, Pompeu CM, Sousa JC, Silva VC, Nunes AA. Fibroma ossificante complicado com piocele de seios paranasais: um relato de caso. Rev Med UFC. 2021;61(1):1-4. 